\title{
An emergency contraception algorithm based on risk assessment: changes in clinicians' practice and patients' choices
}

\author{
Rebecca Jayne McKay, ${ }^{1}$ Lynne Gilbert ${ }^{2}$
}

\begin{abstract}
${ }^{1}$ Subspecialist Registrar in Sexual and Reproductive Healthcare, Department of Reproductive Medicine, Cambridge Universities Hospitals NHS Foundation Trust, Cambridge, UK

${ }^{2}$ Associate Specialist in Sexual and Reproductive Healthcare, Cambridgeshire Community Services NHS Trust, The Laurels CASH Clinic, Cambridge, UK
\end{abstract}

\section{Correspondence to Dr Rebecca Jayne McKay, Department of Reproductive Medicine, Cambridge Universities Hospitals NHS Foundation Trust, Hills Road, Cambridge CB2 OQQ, UK; rjmckay@doctors.org.uk}

Received 18 September 2012 Revised 12 November 2012 Accepted 15 November 2012 Published Online First 21 February 2013
To cite: McKay RJ, Gilbert L. J Fam Plann Reprod Health Care 2013;39:201-206.

\begin{abstract}
Objectives When ulipristal acetate (UPA) was introduced into the Contraceptive and Sexual Health Services service in June 2011 an algorithm was developed to guide method selection. The aim was to encourage clinicians to assess risk and to offer a copper intrauterine device (IUD) as first-line choice with hormonal methods as second-line choice.
\end{abstract}

Methods UPA was the second-line choice for presentations up to 120 hours after high-risk episodes (i.e. around the time of ovulation). Women at lower risk were also offered UPA if presentation was between 72 and 120 hours after unprotected intercourse unless the risk was very low, in which case levonorgestrel $1.5 \mathrm{mg}$ was the second-line choice.

Results Prior to the protocol only $49 \%$ of women were offered an IUD compared with $61.6 \%$ afterwards. This improved further in the subgroup of women at high risk where $93.3 \%$ were offered an IUD compared with 59\% initially. After the introduction of UPA the percentage of women accepting the IUD fell from $30 \%$ to $14.1 \%$. Overall $14.5 \%$ of women received UPA compared to $7.8 \%$ receiving an IUD.

Conclusions Introduction of a standardised protocol for the provision of emergency contraception has significantly increased the proportion of women offered an IUD as postcoital contraception, particularly in women at high risk of conception. Introduction of UPA has seen a halving of the number of women accepting the IUD. This is cause for concern. Further investigation into the reasons behind this decrease in IUD uptake needs to be undertaken.

\section{INTRODUCTION}

Emergency contraception (EC) is indicated for the prevention of pregnancy following unprotected sexual intercourse (UPSI) or a

\section{KEY MESSAGE POINTS}

- Introduction of a risk assessment algorithm for the provision of emergency contraception (EC) increased the offering of the emergency intrauterine device (IUD), particularly amongst those at high risk of conception.

- Introduction of ulipristal acetate as an alternative method of EC has resulted in a reduction in the uptake of the emergency IUD.

- Further education of staff and patients is required to redress this balance.

known or suspected contraceptive failure. The Omnibus Survey 2008/09 for the Office for National Statistics showed that $7 \%$ of women in the UK had used EC in the previous year. ${ }^{1}$ Whilst it is difficult to prove on a population level that EC prevents unintended pregnancy, at an individual level it has been shown to reduce the chance of conception. ${ }^{2-5}$ The most effective method of EC is the copper intrauterine device (IUD), which is estimated to prevent $>99 \%$ of expected pregnancies. ${ }^{6}$ The Faculty of Sexual \& Reproductive Healthcare (FSRH) Clinical Guidance on Emergency Contraception published in August 2011 (updated in January 2012) recommends that all women, in whom it is appropriate, should be offered a copper IUD as a first-line option. ${ }^{7}$ However, many women are unaware of the emergency IUD and the level of awareness has in fact declined over the past decade. The Omnibus Survey for the Office for National Statistics 2000-2001 reported that $49 \%$ of women were aware of the emergency IUD; the comparable figure in the 2008-2009 survey was 40\%, although $91 \%$ of women had heard of oral postcoital contraception. Moreover, fitting an 
emergency IUD is not always practicable and for many women it may be unacceptable or unsuitable. Oral postcoital contraception is therefore an important and essential alternative.

There are currently two oral options licensed for use in the UK. Levonorgestrel (LNG) $1.5 \mathrm{mg}$ (Levonelle ${ }^{\circledR}$, Bayer) was licensed in 1999 and was the only option available until ulipristal acetate (UPA) $30 \mathrm{mg}$ (ellaOne ${ }^{\circledR}$, HRA Pharma) was licensed in the UK in May 2009. UPA is a selective progesterone receptor modulator that binds strongly to the progesterone receptors in target tissues (e.g. uterus, cervix, ovaries and hypothalamus) and exerts tissue-selective agonist, antagonist and partial agonist effects. In the context of EC, both LNG and UPA appear to work primarily by inhibiting or delaying ovulation. ${ }^{8}$ One study showed an effect of UPA on endometrial thickness but the significance of this has not been elucidated. ${ }^{9}$ Whereas LNG is ineffective after the onset of the luteinising hormone (LH) surge, UPA administered between the onset and the peak of the LH surge can delay follicular rupture and appears to be more effective than LNG around the time of ovulation. ${ }^{10}{ }^{11}$ Figure 1 illustrates the 'window of opportunity' for action of the three EC options. A combined analysis of four World Health Organization trials looking at pregnancy rates when LNG is delayed showed no significant differences in the second, third and fourth day with respect to the first day but on the fifth day women were six times more likely to get pregnant. ${ }^{12}$ UPA continues to be effective for up to 120 hours with no decline in efficacy over this time period and is significantly more effective than LNG when $72-120$ hours have elapsed since UPSI. ${ }^{13}$

In June 2011, a 6-month pilot was set up to trial the use of UPA in the Contraceptive and Sexual Health Services (CASH) service based in Cambridge. The local Medicines Management team agreed to restricted use, commenting on the lack of long-term safety data for UPA, the effect on hormonal methods of contraception 'Quick Started' after UPA and the cost implications. The introduction of UPA therefore

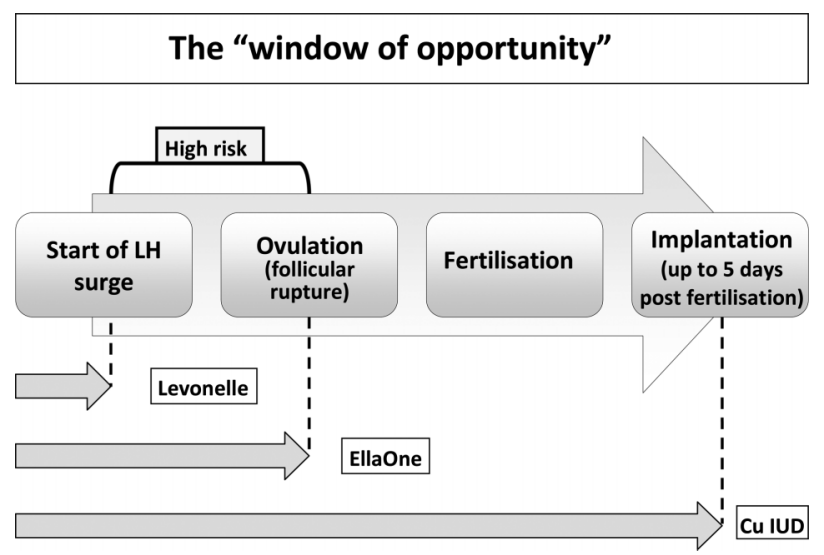

Figure 1 The 'window of opportunity'. Cu IUD, copper intrauterine device; LH, luteinising hormone. presented a challenge to develop a rational basis for selecting an EC method and an opportunity to encourage clinicians to offer an IUD more widely.

The resultant protocol was based on the risk of conception and an algorithm was developed to encourage staff to asses risk from the menstrual, coital and contraceptive history and to offer methods accordingly. Consistent with FSRH recommendations, all women were to be offered an IUD as first-line option. For women declining this option, or in whom it was inappropriate or unsuitable, UPA was offered to two groups of women: (1) those at high risk of conception and (2) all other women presenting between 72 and 120 hours after UPSI unless the risk assessment was low, in which case LNG was offered. UPA was therefore restricted to women who declined an IUD and in whom LNG was likely to have no or minimal effect.

The algorithm was incorporated into a proforma, which included documentation of other FSRH recommendations, namely discussion and/or provision of ongoing contraception and a sexually transmitted infections risk assessment with the offer of screening. A prospective audit was carried out of all EC consultations for the 6 months after introduction of the protocol. EC use had been audited in the previous year and this retrospective audit was used as a comparison. The cost implications to the service of the introduction of UPA were also calculated.

\section{METHODS}

The pilot ran from June 2011 to December 2011. Each presentation for EC was assigned to one of four risk categories: low, high, moderate or indeterminate. The moderate and indeterminate categories were further subdivided into presentation up to 72 hours after UPSI and presentation between 72 and 120 hours. Women who presented following a potential failure of a hormonal method [e.g. overdue depot medroxyprogesterone acetate (Depo-Provera ${ }^{\circledR}$ ) injection and missed oral contraception) were automatically categorised as indeterminate risk. Women not using hormonal contraception were categorised in relation to when in their cycle they had had UPSI. This is illustrated in Figure 2. The likely ovulation date was estimated by subtracting 14 days from the shortest cycle length over the previous 6 months. The reliability of this calculation is questioned by studies that have demonstrated the difficulty in predicting the timing of ovulation; however, it is the accepted method for determining the time limits for safe IUD insertion. High risk was defined as the predicted day of ovulation plus the 3 days before, thus including the days with maximum chance of conception, namely between the onset of the LH surge and ovulation and also the 3 days after ovulation to allow for inaccuracies in the estimated day of ovulation. Low risk was defined as the last 7 days of the cycle and from Day 1 to ovulation minus 8 days. The FSRH guidelines state that the combined pill can be started in the first 


\begin{tabular}{|c|c|c|c|c|c|c|c|c|c|c|c|c|c|c|c|c|c|c|c|c|c|c|c|c|c|c|c|}
\hline \multicolumn{13}{|c|}{ Pre-ovulation } & & \multicolumn{14}{|c|}{ Post-ovulation } \\
\hline 1 & 2 & 3 & 4 & 5 & 6 & 7 & 8 & 9 & 10 & 11 & 12 & 13 & 14 & 15 & 16 & 17 & 18 & 19 & 20 & 21 & 22 & 23 & 24 & 25 & 26 & 27 & 28 \\
\hline \multicolumn{5}{|c|}{ Low } & \multicolumn{5}{|c|}{ Moderate } & \multicolumn{7}{|c|}{ High } & \multicolumn{4}{|c|}{ Moderate } & \multicolumn{7}{|c|}{ Low } \\
\hline
\end{tabular}

Figure 2 Calculating risk of pregnancy.

5 days of the cycle with no extra precautions, suggesting that there is a very low risk of pregnancy during this time period. The remaining days were defined as moderate risk. The algorithm for method selection is illustrated in Figure 3.

Women eligible for UPA on the basis of their risk category had further screening for exclusion criteria (Box 1). Although not excluded within the terms of the product licence, there are very few data on use in women aged under 18 years therefore UPA was not offered to these women. In case of non-attendance, women choosing an IUD were also given LNG if the procedure was not scheduled for the same day.

Prior to the introduction of the proforma, the protocol and supporting documentation was circulated to all clinical staff followed by a face-to-face training session.

The notes of all EC consultations in the 6-month period from June 2011 to December 2011 were analysed 2 months after the end of the study period to allow time to identify patients re-attending following failure of EC. The data extracted included patient demographics, indications for EC, risk category assignment, method of EC provided, future contraception plans and provision. All risk category assignments were checked for accuracy from the menstrual and coital information recorded and any subsequent attendances indicating a failure of EC were noted. The data were then compared with a retrospective audit conducted over 2 months in 2010 prior to the introduction of the algorithm and UPA. At this time there was no structured proforma to guide clinicians on record keeping. There were $345 \mathrm{EC}$ requests from 317 unique patients in the 6 months of the 2011 study and 136 requests in the 2 months of the 2010 study. All case notes were analysed.

\section{RESULTS}

The patient demographics were broadly similar (Table 1). The commonest age range for requesting EC was between 16 and 24 years, with over $60 \%$ of consultations falling in this bracket. The indications for EC were also similar across both datasets with UPSI and condom failure accounting for over $85 \%$ of consultations (Table 1). In the first audit, risk categories were assigned retrospectively by the authors from the information given in the notes. Risk categories after the introduction of the algorithm were specifically recorded by the clinician seeing the patient (Table 1). These were checked by the authors against the menstrual, coital and contraceptive history recorded on the proforma. The assignment was found

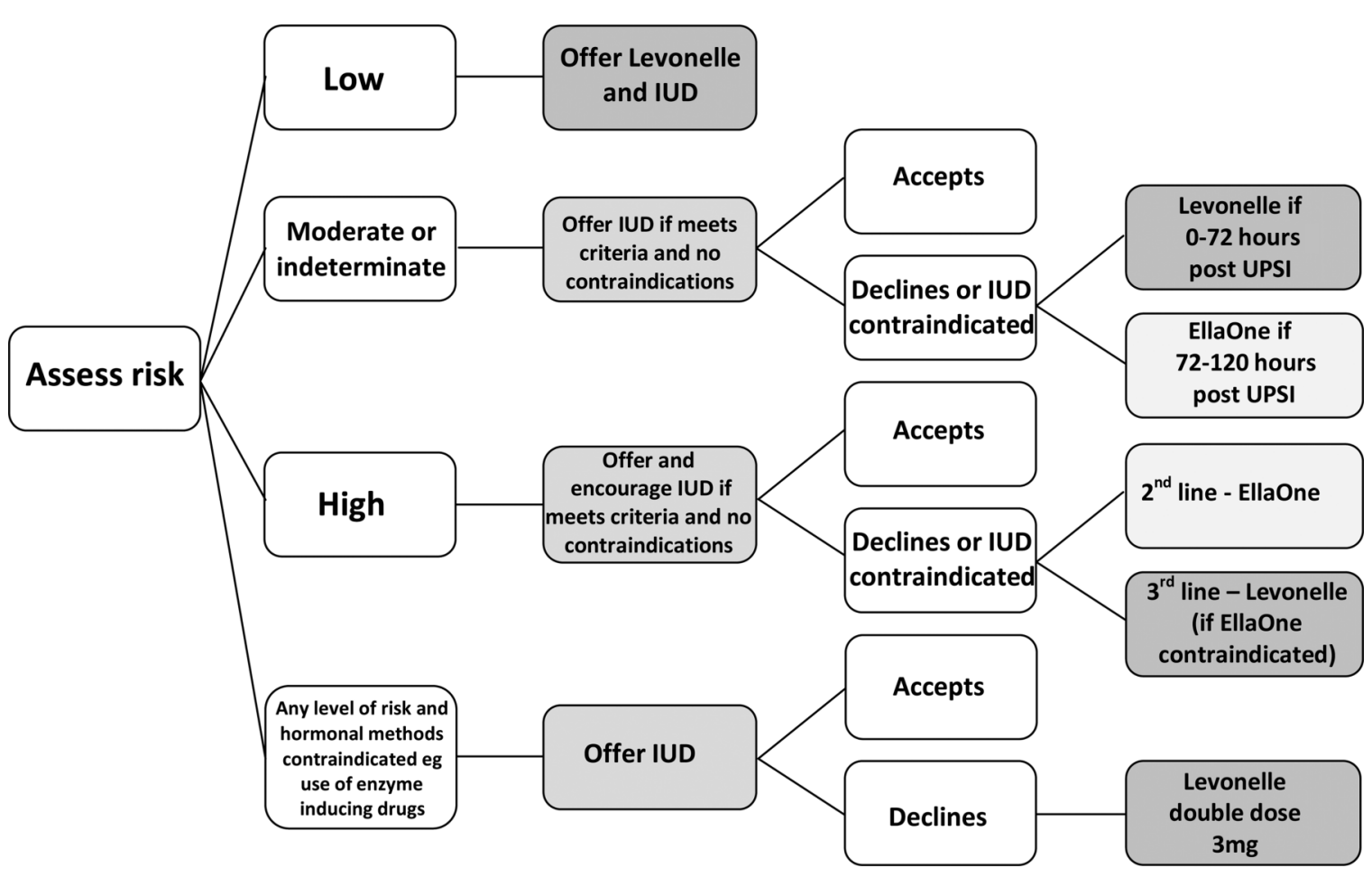

Figure 3 The emergency contraception algorithm. IUD, intrauterine device; UPSI, unprotected sexual intercourse. 


\section{Box 1 Exclusion criteria for ulipristal acetate.}

Women aged under 18 years

- Women on the following interacting drugs:

Enzyme inducer (currently or within the last 28 days)

Antacids

- Proton-pump inhibitors

- $\mathrm{H}_{2}$ receptor antagonists

Ketoconazole/itraconazol/clarithromycin

- Severe asthma

- Galactose intolerance/Lapp lactase deficiency/glucose-galactose malabsorption

- Severe liver or renal disease

- Used levonorgestrel or ulipristal acetate in this cycle

to be incorrect in $8.1 \%(28 / 345)$ of cases. In the very small numbers of incorrect assignment of risk the practitioners tended to err on the side of caution.

We looked at the patients in whom an IUD was deemed to be appropriate. In the first audit $72.8 \%$ (99/136) were eligible for an IUD compared with $89.9 \%(310 / 345)$ afterwards. This greater proportion may be because the algorithm made it easier to be confident of excluding the possibility of an implanted pregnancy and therefore decide that an IUD was safe.

From the data recorded the number of women eligible for an IUD and the number in the subgroup who were also eligible for UPA was established. Prior

Table 1 Patient demographics, indication for emergency contraception and risk categories

\begin{tabular}{|c|c|c|}
\hline Parameter & $\begin{array}{l}\text { Retrospective audit } \\
2010(\%)\end{array}$ & $\begin{array}{l}\text { Post-protocol audit } \\
2011(\%)\end{array}$ \\
\hline \multicolumn{3}{|l|}{ Patient age (years) } \\
\hline$<16$ & $13(9.6)$ & $18 \quad(5.2)$ \\
\hline $16-19$ & $47(34.5)$ & $113(32.7)$ \\
\hline $20-24$ & $38(30.0)$ & $119(34.5)$ \\
\hline $25-29$ & $15(11.4)$ & $48(13.9)$ \\
\hline $30-34$ & $12(8.8)$ & $25(7.2)$ \\
\hline $35-39$ & $5(3.8)$ & $13(3.8)$ \\
\hline $40-44$ & $4 \quad(2.9)$ & $6(1.7)$ \\
\hline $45-49$ & $2(1.4)$ & $3(0.9)$ \\
\hline \multicolumn{3}{|c|}{ Indication for emergency contraception } \\
\hline $\begin{array}{l}\text { Unprotected sexual } \\
\text { intercourse }\end{array}$ & $87(63.9)$ & $176(51.0)$ \\
\hline Condom failure & $29(21.3)$ & $124(36.0)$ \\
\hline $\begin{array}{l}\text { Missed/late pills, } \\
\text { patch or ring }\end{array}$ & $16(11.8)$ & $33(9.6)$ \\
\hline Other & $4^{*}(2.9)$ & $12+(3.5)$ \\
\hline \multicolumn{3}{|l|}{ Risk categories } \\
\hline Low & $28(20.6)$ & $92(26.6)$ \\
\hline Moderate & $34(25.0)$ & $60(17.4)$ \\
\hline High & $42(30.9)$ & $75(21.7)$ \\
\hline Indeterminate & $32(23.5)$ & $118(34.2)$ \\
\hline
\end{tabular}

${ }^{*}$ Combined oral contraceptive and antibiotics (3), late Depo-Provera ${ }^{\circledR}(1)$. tIntrauterine device expulsion (2), late Depo-Provera (3), overdue intrauterine system (2), failure to use extra precautions with 'Quick Start' (3) not recorded (2). to introducing the algorithm, only 49\% (49/99) of women eligible for an IUD were offered one. Although this increased significantly to $61.6 \%$ $(191 / 310)$ after the introduction of the algorithm, this still falls short of the FSRH target of 100\%. However, in the subgroup of women in the high-risk category the improvement was even more marked with only $59 \%(25 / 42)$ offered an IUD prior to the algorithm but over 93\% (70/75) afterwards.

Critically it is the rate of uptake that is important. Before the introduction of risk assessment and UPA, $30 \%(15 / 49)$ of those who were offered an IUD accepted it, resulting in 15\% (15/99) of women for whom an IUD was appropriate having the device fitted. After introduction of the algorithm only $14.1 \%$ (27/191) of those offered accepted the IUD and this represented $8.7 \%(27 / 310)$ of those eligible and therefore a $42 \%$ fall in the rate of acceptance. For women in the high-risk category there was a smaller (20\%) drop in acceptance of the IUD after the introduction of the algorithm (14/70 vs 10/25). Overall $18.7 \%$ $(14 / 75)$ of those at high risk had an IUD afterwards compared with $24 \%(10 / 42)$ before. This is very disappointing given the improvement in the rate of offering an IUD but perhaps not that surprising given the alternative of UPA. These data are summarised in Figure 4. Of the women who were eligible for both UPA and an IUD, 79\% chose UPA with only 17\% choosing an IUD and 4\% preferred LNG.

In the cohort of patients after introduction of the algorithm, there were four (1.2\%) confirmed pregnancies, all of whom had had LNG. None of these women were assessed as high risk. Two of the women were eligible for an IUD and were offered it appropriately but declined. The other two were ineligible for both UPA and an IUD as the possibility of an early undetected implanted pregnancy could not be excluded.

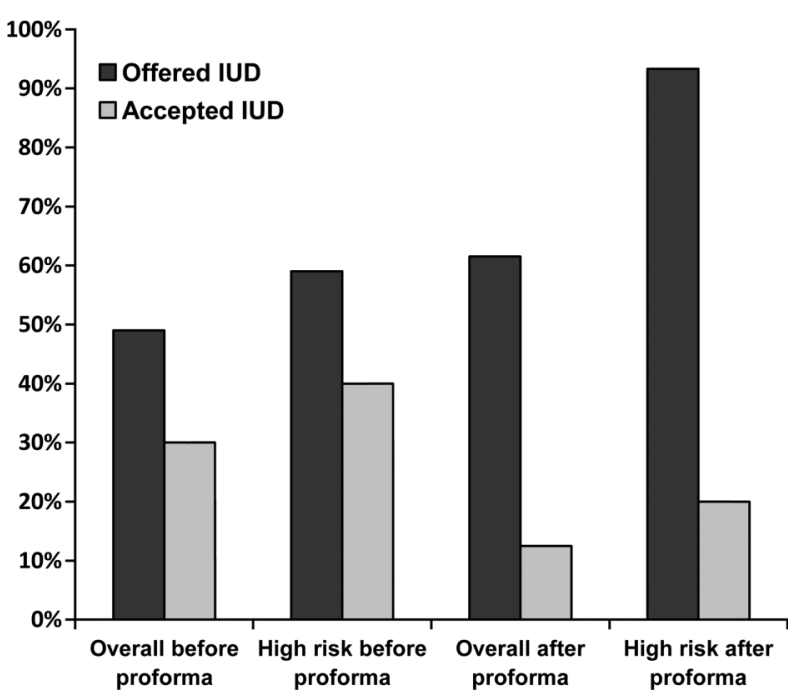

Figure 4 Emergency intrauterine device (IUD) before and after the introduction of ulipristal acetate and the risk assessment proforma. 
UPA cost our service $£ 16.95$ per dose while LNG cost $£ 5.37$. In the 6 -month period UPA was provided 50 times at a drug cost of $£ 847.50$. Had all these patients received LNG instead the excess cost of providing UPA would have been $£ 579$. However, this assumes that all those accepting UPA would previously have used LNG rather than an IUD. An IUD costs $£ 8-£ 11$ but usually requires another appointment with a trained fitter, plus other IUD fitting costs. The cost of fitting an IUD is therefore greater than the difference in cost between LNG and UPA. It is difficult to ascertain whether UPA has cost the service money over 6 months as there have been proportionally fewer IUD fittings. A cost effectiveness analysis comparing UPA vs LNG $1.5 \mathrm{mg}$ for the prevention of unintended pregnancy was undertaken by Cameron et al. and showed that UPA was a cost-effective alternative as the additional costs of preventing an unintended pregnancy exceed the additional drug costs. ${ }^{14}$ This looked at the National Health Service (NHS) as a whole and did not take into account the additional costs of providing UPA to an individual service. This is increasingly important in the modern day fragmented NHS.

\section{DISCUSSION}

The use of a structured proforma that encourages risk assessment and an algorithm for method selection has made clinicians more aware of the superior efficacy of the emergency IUD compared to the oral options. The rate of offering the IUD has increased accordingly. Retrospective audits in this service have shown a year-on-year increase in offering the IUD from $22 \%$ in 2007 to $61.5 \%$ in 2011 . Unfortunately, since the introduction of UPA there has been a decrease in women's acceptance of the IUD. As the IUD remains the most effective method of EC and is the only option that works after ovulation, a reduction in uptake may lead to an increase in the number of unplanned pregnancies.

It is difficult to establish why the introduction of UPA led to a reduction in IUD acceptance but it is likely to be a combination of patient and clinician factors. A small study in London looked at the reasons women give for choosing oral EC rather than the IUD. ${ }^{15}$ This showed that most women had unrealistic expectations as to how effective the postcoital contraceptives were and a lack of knowledge about the IUD. An oral method is undoubtedly easier not only for the patients but also for the clinicians who have to arrange for the device to be fitted and for those who perform the procedure. Against this background one could surmise that UPA has conveniently provided a more effective alternative to the IUD than LNG and has been sold as such, without sufficiently emphasising the superiority of the IUD over both oral methods.

An anonymous survey of clinicians to establish perceived barriers and attitudes to presenting the option of an emergency IUD and performing the procedure is planned. This will inform further education of CASH staff prior to the audit being repeated.

Whilst the reduction in IUD uptake is disappointing, the level of acceptance of IUD is high compared to other services. A study in a CASH service in Edinburgh showed that only $2 \%$ of the women attending for EC accepted an IUD compared with $8.7 \%$ in Cambridge, even after the introduction of UPA. ${ }^{16}$ Summary statistics (KT 31 data) showed that emergency IUD uptake was only $3 \%$ in community clinics nationally. ${ }^{17}$ Educating both women and clinicians is essential to improving the acceptance of an IUD as postcoital contraception.

It is possible to criticise the algorithm developed as it is based on assessing the risk of conception. This is notoriously difficult as it is dependent on a woman's accurate knowledge of her own menstrual cycle, when ovulation is likely to occur and specific information about the timing of all episodes of UPSI. Without some assessment, however, it is not possible to guide choices and establish eligibility for an IUD. However, this process is essential to inform the woman and to determine whether she is eligible for the IUD. An algorithm makes the process more robust and ensures consistency of approach between clinicians within the service. Only $8 \%$ of those women seen after introduction of the algorithm had an inaccurate risk assessment. Unless use of UPA is unrestricted, such an algorithm ensures it is offered to those likely to benefit the most.

In summary, the authors believe that the introduction of a standardised algorithm for the provision of EC leads to more women being offered the IUD, and with further education of staff and patients should lead to an increased acceptance of the IUD.

Acknowledgements The authors would like to thank the nurses and doctors of the Laurels CASH service for completing the proformas.

Funding None.

Competing interests None.

Ethics approval This study was granted ethical approval by the Cambridge Universities NHS Foundation Trust.

Provenance and peer review Not commissioned; externally peer reviewed.

\section{REFERENCES}

1 Lader D. Opinions Survey Report No. 41. Contraception and Sexual Health, 2008/09. 2009. http://www.ons.gov.uk/ons/rel/ lifestyles/contraception-and-sexual-health/2008-09/2008-09.pdf [accessed 8 November 2012].

2 Raymond EG, Trussell J, Polis CB. Population effect of increased access to emergency contraception pills. Obstet Gynaecol 2007;109:181-188.

3 Cheng L, Che Y, Gulmezoglu AM. Interventions for emergency contraception. Cochrane Database Syst Rev 2012;8:CD001324.

4 Raymond E, Taylor D, Trussell J, et al. Minimum effectiveness of the levonorgestrel regimen of emergency contraception. Contraception 2004;69:79-81. 
5 Glasier AF, Cameron ST, Logan SJS, et al. Ulipristal acetate versus levenorgestrel for emergency contraception: a randomized non-inferiority trial and meta-analysis. Lancet 2010;375:555-562.

6 Cleland K, Zhu H, Goldstruck N, et al. The efficacy of intrauterine devices for emergency contraception: a systematic review of 35 years of experience. Hum Reprod 2012;85:1994-2000.

7 Faculty of Sexual \& Reproductive Healthcare Clinical Effectiveness Unit. Emergency Contraception. 2012. http:// www.fsrh.org/pdfs/CEUguidanceEmergencyContraception11. pdf [accessed 8 November 2012].

8 Gemzell-Danielsson K. Mechanism of action of emergency contraception. Contraception 2010;82:404-409.

9 Stratton P, Levens ED, Hartog B, et al. Endometrial effects of a single early luteal dose of the selective progesterone receptor modulator CDB-2914. Fert Steril 2010;93:2035-2041.

10 Brache V, Cochon L, Jesam C, et al. Immediate pre-ovulatory administration of $30 \mathrm{mg}$ ulipristal acetate significantly delays follicular rupture. Hum Reprod 2010;25:2256-2263.

11 Noe G, Croxatto HB, Salvatierra AM, et al. Contraceptive efficacy of emergency contraception with levonorgestrel given before or after ovulation. Contraception 2010;81:414-420.

12 Piaggo G, Kapp N, von Hertzen H. Effect on pregnancy rates of the delay in administration of levenorgestrel for emergency contraception: a combined analysis of four WHO trials. Contraception 2011;84:35-39.

13 Creinin MD, Schlaff WD, Archer DF, et al. Progesterone receptor modulator for emergency contraception: a randomized controlled trial. Obstet Gynaecol 2006;108: 1089-1097.

14 Thomas CM, Schmid R, Cameron S. Is it worth paying more for emergency hormonal contraception? The cost effectiveness of ulipristal acetate versus levonorgestrel $1.5 \mathrm{mg}$. J Fam Plann Reprod Health Care 2010;36:197-201.

15 Bharadway P, Saxton JC, Mann SN, et al. What influences young women to choose between the emergency contraceptive pill and an intrauterine device? A qualitative study. Eur J Contracept Reprod Healthcare 2011;16:201-209.

16 Cameron ST, Glasier A, Johnstone A, et al. Ongoing contraception after use of emergency contraception from a specialist contraceptive service. Contraception 2011;84: 368-371.

17 Statistical Services Contraception 1997-2008 The NHS Information Centre KT31 Return. Table 6: Number of contacts for emergency contraceptives at NHS community contraception clinics, by type, 1997/98 to 2011/12. http:// data.gov.uk/dataset/nhs-contraceptive-services-england-201112-community-contraceptive-clinics [accessed 8 November 2012]. 